\title{
Advanced 3D and Live Imaging Reveals Phenotypic Consequences of Disruptions in Mechanical and Genetic Mechanisms Underlying Embryonic Cardiovascular Development
}

\author{
Mary E. Dickinson ${ }^{1}$ \\ 1. Department of Molecular Physiology and Biophysics and Molecular and Human Genetics, Baylor \\ College of Medicine, Houston, TX, USA.
}

The Dickinson lab combines expertise in mouse genetics, developmental biology, imaging and bioengineering to focus on understanding birth defects of the cardiovascular system and to determine how mechanical programs influence the genetic programs that guide mammalian development. Toward this goal, the Dickinson lab utilizes live cell and intravital imaging using confocal, multiphoton, Optical Coherence Tomography, Optical Projection Tomography, microCT and Lightsheet microscopy to study heart development, angiogenesis and vessel remodeling. As part of the NIH-funded Knockout Mouse Project at BCM, the Dickinson lab has recently developed a state-of-the-art, high throughput phenotyping pipeline to characterize structural defects in lethal alleles of knock out mice. Recessive lethal mutations reveal a rich resource of disease alleles and insights into these essential genes has been recently described (Dickinson et al 2016).

Our previous work has used live embryo imaging and confocal and lightsheet microscopy to determine forces exerted by early blood flow in the embryo (Jones et al 2004) and to show that vascular remodeling of the mouse embryo yolk sac does indeed depend on viscous forces emanating from blood flow (Lucitti et al 2007). Live imaging revealed that directed endothelial migration and vessel wall fusion are responsible for outward remodeling and revealed distinct behaviors on the arterial and venous sides (Udan et al 2013).

Insights to the molecular mechanisms required for vascular remodeling are being revealed using mouse mutants with disrupted vascular remodeling. New mutants have been discovered via high throughput analysis of engineered null mouse mutations (Dickinson et al 2016) using state-of-the-art microCT imaging methods (Hsu et al 2016) [6].

\section{References:}

[1] Jones EA et al, Genesis. 34(4) (2002), p. 228.

[2] Lucitti JL et al, Development. 134(18) (2007), p. 3317.

[3] Udan, R.S. et al, Development 22 (2014), p. 4406.

[4] Dickinson, ME et al, Nature 537 (2016), p. 508.

[5] Hsu C-W et al, Dev Biol. (2016).

[6] The authors acknowledge funding from the NIH UM1HG006348, R01 HL120140 and

R01HL128064. 
Figure 1. Early blood circulation in an E8.5 mouse embryo. GFP-labeled blood cells reveal the paths of blood flow within the early embryo.

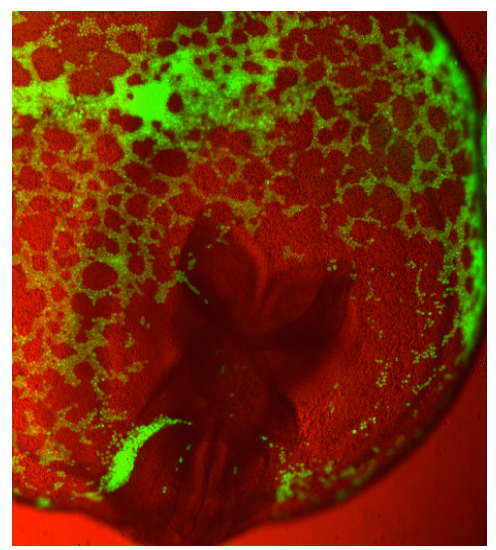

Figure 2. MicroCT image of an early post-implantation (E9.5) mouse embryo showing the detailed vessel remodeling in the yolk sac. Mutant embryos can be screened by microCT to define genes required for normal vessel remodeling.

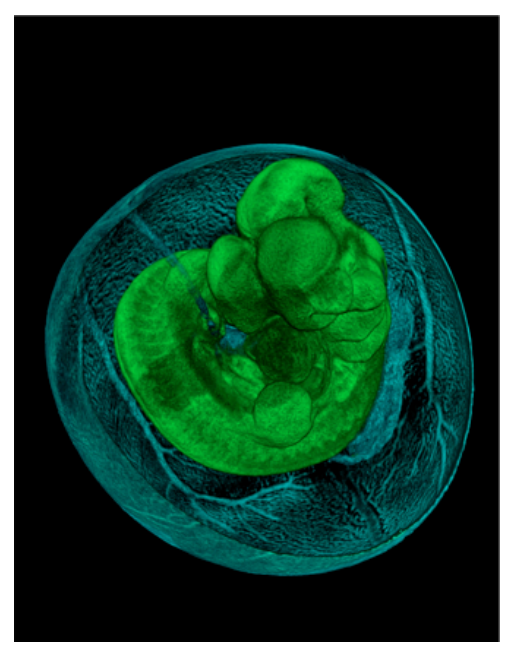

